УДК 616.24-002-02:616.24-036-12

DOI

\title{
ВНЕБОЛЬНИЧНАЯ ПНЕВМОНИЯ У ПАЦИЕНТОВ С ХОБЛ: ВРЕМЕННАЯ КОМОРБИДНОСТЬ ИЛИ ФАКТОР РИСКА ЛЕТАЛЬНОГО ИСХОДА?
}

\section{COMMUNITY-ACQUIRED PNEUMONIA IN PATIENTS WITH COPD: TEMPORARY COMORBIDITY OR RISK FACTOR FOR DEATH?}

\author{
О.М. Урясьев, Ю.А. Панфилов, Я.Н. Гринькова, М.А. Гранаткин, А.А. Пыко \\ O.M. Uryasyev, Yu.A. Panfilov, Y.N. Grinkova, M.A. Granatkin, A.A. Pyko
}

Рязанский государственный медицинский университет имени академика И.П. Павлова, Россия, 390026, г. Рязань, ул. Высоковольтная, 9

Ryazan State Medical University, 9 High Voltage St, Ryazan, 390026, Russia

E-mail: panfilov.rzgmu@gmail.com

\begin{abstract}
Аннотация
В настоящее время внебольничная пневмония занимает одно из лидирующих мест среди часто встречающихся инфекционных болезней. ХОБЛ считают фактором риска для развития внебольничной пневмонии, и многочисленные работы и исследования, посвященные изучению внебольничной пневмонии (ВП), показали, что ХОБЛ упоминается как сопутствующая патология у 19-62 \% больных с ВП. Что касается статистики о развитии внебольничной пневмонии у больных с обострением ХОБЛ, то в настоящее время напечатано незначительное количество работ, посвященных данной проблеме. Большинство этих исследований посвящены клиническим случаям с тяжелым обострением ХОБЛ (с картиной острой дыхательной недостаточности). По данным международной статистики, смертность больных с ХОБЛ госпитализированных с обострением в стационар составляет - $8 \%$, через год после рецидива $-23 \%$. Если же говорить о больных с ОДН на фоне обострения ХОБЛ, то смертельный исход составляет $24 \%$, у пациентов старше 65 лет - $30 \%$.
\end{abstract}

\begin{abstract}
At present, community-acquired pneumonia (CAP) is one of the leading places among common infectious diseases. Chronic constructive pulmonary disease is considered a risk factor for the development of community-acquired pneumonia, and numerous studies and studies on the study of community-acquired pneumonia have shown that COPD is mentioned as a concomitant pathology in 19-62\% of patients with CAP. As for statistics on the development of community-acquired pneumonia in patients with exacerbation of COPD, a small number of works on this problem are currently published. Most of these studies are devoted to clinical cases with severe exacerbation of COPD (with a picture of acute respiratory failure). According to international statistics, the mortality rate of patients with chronic obstructive pulmonary disease hospitalized with exacerbation to the hospital is $8 \%$, a year after the relapse $-23 \%$. If we talk about patients with acute respiratory failure amid exacerbation of COPD, then the death rate is $24 \%$, in patients older than 65 years $-30 \%$.
\end{abstract}

Ключевые слова: внебольничная пневмония, хроническая обструктивная болезнь легких (ХОБЛ), фактор риска летального исхода.

Keywords: community-acquired pneumonia, chronic obstructive pulmonary disease (COPD), death risk factor.

\section{Введение}

В настоящее время внебольничная пневмония (ВП) занимает одно из лидирующих мест среди часто встречающихся инфекционных болезней. По имеющимся данным, в РФ 
в 2017 г. зафиксировано 412,3 случая ВП на 100 тыс. населения, что сравнимо с 2016 г. 418,02 случая ВП на 100 тыс. населения и на $22 \%$ выше, чем в 2015 году $(337,1)$, и была максимальной за последние 5 лет (в 2012 году - 345,0; 2013 г. - 389,2; 2014 г. - 349,5). Больше всего зафиксировано в Дальневосточном и Уральском федеральных округах: 560,84 и 503,31 на 100 тыс. населения. Каждый год отмечается постепенное повышение этого показателя [Биличенко, Чучалин, 2018].

Смертность при ВП у пациентов среднего и молодого возраста без наличия сопутствующих заболеваний составляет приблизительно 1-3\%. А у лиц старше 60 лет с наличием у них сопутствующей патологии показатель летальности - 15-30\%. В 2016 г. РФ смертность от пневмонии составила 30716 случаев [Биличенко, Чучалин, 2018; Внебольничная пневмония: клинические рекомендации, 2018].

Как и при любом другом заболевании, при оценке ВП важное значение имеет выявление факторов риска, определяющих ключевые параметры клинического течения и прогноза заболевания, включая летальность. К наиболее изученным и значимым модифицируемым факторам риска традиционно относят статус курильщика, сопутствующая терапия (прием глюкокортикоидов, нестероидных противовоспалительных средств, антисекреторных препаратов и т. д.), сопутствующие заболевания, включая хроническую сердечную недостаточность, сахарный диабет, алкоголизм, наркоманию и др. Определенный интерес представляет и такое сочетание, как комбинация ХОБЛ и ВП [Чучалин и др., 2016; Ефремова и др., 2016].

Подсчитано, что число случаев ХОБЛ в 2010 году составило 384 миллиона, с глобальной распространенностью 11,7\%. Во всем мире ежегодно происходит около трех миллионов смертей вследствие ХОБЛ. По данным [GOLD, 2019], в течение 30 лет ожидается рост распространенности ХОБЛ в связи с увеличением частоты встречаемости курящего населения в странах, и к 2030 году и ежегодно от ХОБЛ и сопутствующих патологий уровень смертности может составлять $\geq 4,5$ млн людей.

По данным зарубежных источников, при госпитализации пациента в стационар по поводу обострения ХОБЛ смертность достигает $8 \%$, а в течение последующего года $23 \%$. При этом если обострение ХОБЛ сопровождается значимой дыхательной недостаточностью, то риск развития летального исхода увеличивается до $24 \%$, а при наличии такого отягощающего фактора, как возраст более 65 лет, - до $30 \%$. [Groenewegen et al., 2003; Seneff et al., 1995].

Таким образом, существует ряд моментов, позволяющих отнести ХОБЛ к клинически значимым состояниям у пациентов с ВП, среди которых наибольшую роль играет высокая встречаемость ХОБЛ среди населения, высокий процент пациентов с коморбидной патологией, тяжелое течение заболевания, возрастающая антибиотикорезистентность, сложности диагностики ВП у больных ХОБЛ на фоне обострения, а также морфологические и функциональные изменения в респираторной системе [Дворецкий, 2015].

\section{Эпидемиология ВП при ХОБЛ}

В настоящее время есть ряд работ, отражающих проблему исследования сопутствующей патологии при ВП, однако данные о сочетании ВП с ХОБЛ довольно ограничены [Кузубова и др., 2014]. При этом по данным некоторых эпидемиологических исследований ХОБЛ является одной из наиболее часто встречающихся патологией у пациентов с пневмонией.

На риск госпитализации при пневмонии у больных с ХОБЛ влияет ряд параметров, включая увеличение тяжести течения заболевания, стаж и интенсивность курения, показатели функции внешнего дыхания. Например, по результатам исследования [Mannino et al., 2009] за 20375 пациентами в возрасте 45 лет и старше, у людей с нормальной функцией легких был самый низкий показатель госпитализации по поводу пневмонии (1,5 на 1000 человеко-лет), а самый высокий данный показатель - у больных ХОБЛ III и IV стадией 22,7 на 1000 человеко-лет. Тяжесть течения ХОБЛ является важным и независимым пре- 
диктором госпитализации по поводу пневмонии в этой когорте исследования. В 2012 году опубликованы результаты проспективного наблюдения за группой пациентов с ХОБЛ старше 45, в ходе которого было показано, что заболеваемость ВП среди исследуемой группы составила 22,4 случая на 1000 человеко-лет и значительно увеличивается в возрасте 65 лет и старше [Müllerova et al., 2012]. Как результат, исследователи отнесли возраст 65 лет и старше, наличие сопутствующих заболеваний, таких как застойная сердечная недостаточность и деменция, к независимым предикторам ВП. Предыдущие тяжелые обострения ХОБЛ, требующие госпитализации, и тяжелая ХОБЛ, требующая домашнего кислородного или небулайзерного лечения, также были в значительной степени связаны с риском развития ВП [Коррейа и др., 2013].

В одной испанской работе, включавшей 596 пациентов, общая заболеваемость ВП составила 55,1 на 1000 человеко-лет. В течение 3 лет наблюдения как минимум 1 эпизод пневмонии развился у 75 (12,6 \%) пациентов. Всего зарегистрировано 88 эпизодов ВП на 75 пациентов. Тяжесть ХОБЛ, оцененная по ОФВ1, была легкой у 9 пациентов, средней у 24 и тяжелой - у 42.76 эпизодов пневмонии (86,3\%) расценены как ВП, а 12 (13,6 \%) как нозокомиальные. Общая смертность составила 12,5 \% (11/88), среди которых внутрибольничная пневмония обусловила 41,7 \% (5/12) летальных случаев, а ВП - 7,8 \% (6/76) случаев. Смертность при ВП зарегистрирована у пациентов 4 и 5 степени тяжести ВП, при меньшей тяжести ВП летальных исходов выявлено не было.

Полученные данные согласуются с результатами других авторов: встречаемость на уровне 78,5 на 1000 человеко-лет за 3-летний период наблюдения и летальность в 18,7 \% случаев [Баймакова и др., 2009], при этом вероятность развития ВП увеличивалась с возрастом, по мере уменьшения индекса массы тела и наличия другой сопутствующей патологии (онкопатология бронхолегочной системы, бронхэктазы) и терапии (ингаляционные кортикостероиды) [Lieberman et al., 2002; Lin et al., 2013].

Патолого-анатомические исследования также подтверждают высокую частоту ВП у пациентов с ХОБЛ. По результатам проведенного анализа протоколов аутопсии и историй болезней умерших больных с хроническими заболеваниями органов дыхания пожилого и старческого возраста [Игнатьев и др., 2008] ВП посмертно диагностирована у 46,5 \% умерших пациентов с ХОБЛ. В 66,67 \% случаев ВП явилась основным заболеванием, в $33,33 \%$ - сопутствующим или фоновым. У других авторов [Черняев, 2005] посмертная диагностика ВП у больных с ХОБЛ еще выше и достигает 70,9\%.

Таким образом, сочетание ХОБЛ и ВП в пожилом и старческом возрасте следует рассматривать коморбидность с взаимным отягощением. С одной стороны, пневмония как грозное осложнение для пациентов пожилого возраста может определить неблагоприятный исход заболевания, а с другой - приводит к быстрой декомпенсации ХОБЛ и летальному исходу.

\section{Особенности течения ВП при ХОБЛ}

Характерными особенностями тяжелой ВП при ХОБЛ являются проявления полиорганной недостаточности, в том числе дыхательной, и возможная генерализация инфекции.

Несколько исследований показали, что риск фатального исхода при тяжелой ВП можно предсказать по клинико-инструментальным и лабораторным критериям течения ВП (см. табл.). В исследовании американских ученых [Farr et al.,1991] риск смертельного исхода повышается в 20 раз у больных с ВП, имеющих признаки: ЧДД 30 в мин и более, диастолическое артериальное давление 60 мм рт.ст. или менее и азот мочевины крови более 7 ммоль/л, в отличие от пациентов без перечисленных признаков.

Ученые Германии [Ewig et al., 1995] в своем многомерном анализе показали, что такие показатели исследования, как ЧСС, систолическое АД и уровень ЛДГ в сыворотке крови были тесно связаны с летальным исходом. Таким образом, у пациентов, имеющих 2 из трех показателей: частота сердечных сокращений $\geq 90$ уд/мин, систолическое $\mathrm{AД} \leq 80$ мм рт. ст., уровень ЛДГ $\geq 260$ Ед/л, повышается риск смерти в 6 раз. 
Критерии тяжелого течения внебольничной пневмонии

Criteria for severe community-acquired pneumonia

\begin{tabular}{|c|l|l|}
\hline & \multicolumn{1}{|c|}{ Клинико-инструментальные } & \multicolumn{1}{|c|}{ Лабораторные } \\
\hline 1 & $\begin{array}{l}\text { Острая дыхательная недостаточность: } \\
\text { ЧДД > 30 в минуту; } \\
\text { SаО2 <90\% }\end{array}$ & Лейкопения $(<4 \times 109 /$ л) \\
\hline 2 & $\begin{array}{l}\text { Гипотензия: } \\
\text { Систолическое АД <90 мм.рт.ст. } \\
\text { Диастолическое АД <60 мм.рт.ст. }\end{array}$ & $\begin{array}{l}\text { Гипоксемия: } \\
\text { РО2 }<60 \text { мм.рт.ст. }\end{array}$ \\
\hline 3 & Двух- или многодолевое поражение легких & Гемоглобин < 100 г/л \\
\hline 4 & Нарушение сознания & Гематокрит $<30 \%$ \\
\hline 5 & $\begin{array}{l}\text { Внелегочный очаг инфекции (менингит, } \\
\text { перикардит и др.) }\end{array}$ & $\begin{array}{l}\text { Острая почечная недостаточность: } \\
\text { Креатинин крови }>176,7 \text { мкмоль/л; } \\
\text { Азот мочевины }>7,0 \text { ммоль/л }\end{array}$ \\
\hline 6 & Анурия & \\
\hline
\end{tabular}

Результаты о неблагоприятном исходе были получены также в 2-х других исследованиях у пациентов с ВП на фоне ХОБЛ. По данным ретроспективного исследования [Restrepo et al., 2006], у пациентов с ХОБЛ, госпитализированных с ВП, наблюдалась более высокая 30-дневная (отношение рисков 1,32) и 90-дневная смертность (отношение рисков 1,34$)$, чем у пациентов без ХОБЛ.

Во вторичном анализе проспективного исследования [Rello et al., 2006] у больных ХОБЛ летальность в ОИТ (отношение шансов (Ош) 1,58$)$ и необходимость искусственной вентиляции легких (Ош 2,78) были выше, чем у пациентов без ХОБЛ. Следовательно, пациенты с ХОБЛ, госпитализированные с ВБ в отделение интенсивной терапии, имели более высокую летальность и потребность в искусственной вентиляции легких по сравнению с пациентами без ХОБЛ.

Исходя из результатов исследований [Restrepo et al., 2006] и [Rello et al., 2006], было предложено включить сопутствующее состояние ХОБЛ предиктором повышенной смертности у пациентов, госпитализированных по поводу ВП.

В 2013 был проведен еще один мета-анализ 24 результатов исследований: в 13 исследованиях статистика показала незначительный риск смертности, связанный с сопутствующей ХОБЛ и ВП, по сравнению с ВП без ХОБЛ в пяти исследованиях. В оставшихся исследованиях взаимовлияние двух данных нозологий было оценено как неопределенное [Loke et al., 2013].

Неблагоприятный прогноз у больных с ВП на фоне ХОБЛ обусловлен частым развитием острой дыхательной недостаточности/декомпенсацией сердечно-сосудистой патологии. По некоторым результатам ВП является причиной острой дыхательной недостаточности в $15 \%$ случаев у больных ХОБЛ. В других исследованиях данный показатель еще выше и может достигать $36 \%$ [Авдеев и др., 2006].

Причиной развития острой дыхательной недостаточности может быть как острое поражение альвеолярно-капиллярного пространства при ВП, так и утяжеление уже имеющейся хронической дыхательной недостаточности, характерной для ХОБЛ. Поэтому рассматриваемая коморбидность характеризуется более высокой вероятностью необходимости более тщательного мониторинга пациентов с возможной госпитализацией в ОРИТ, адекватного назначения антибактериальной терапии, ИВЛ и др.

Ухудшение газообмена может приводить к декомпенсации сопутствующих заболеваний, что также увеличивает летальность. Так, наличие в анамнеза патологии сердечнососудистой системы, особенно осложненной хронической сердечной недостаточностью, ведет к ухудшению прогноза и увеличению смертности [Sibila, 2014]. 


\section{Диагностика: ВП или обострение ХОБЛ?}

Дифференциальный диагноз инфекционного обострения ХОБЛ и ВП остается сложным вопросом практического здравоохранения, актуальным как для врачей терапевтического профиля, так и для специалистов-пульмонологов. При этом возможна как гипо-, так и гипердиагностика рассматриваемых состояний. С практической точки зрения точная и своевременная верификация ВП у пациентов с обострением ХОБЛ является важным моментом не только для своевременной диагностики, но и для назначения адекватной терапии.

Согласно данным патолого-анатомических вскрытий, ВП часто пропускается прижизненно при наличии ХОБЛ, что может быть связано как с общим увеличением количества соматических заболеваний, так и взаимном модифицировании и стирании клинических проявлений. По данным некоторых авторов, ВП прижизненно пропускается в $35 \%$ случаев стационарного нахождения пациентов и до $82 \%$ поликлинических ситуаций [Черняев, 2005]. При этом на частоту витальной диагностики ВП именно ХОБЛ оказывает максимальное отрицательное влияние среди всей возможной сопутствующей патологии.

Клиническая картина ХОБЛ может маскировать проявления ВП, что приводит к прижизненной гиподиагностике последней. Вирусная инфекция может предшествовать обеим рассматриваемым патологиям (см. рис.) [Баймакова и др., 2009]. По критериям N. Anthonisen и соавт. (1987) больше выражена тяжесть обострения у больных с ВП $(\mathrm{p}=0,024)$. В ходе исследования отмечено более значимое повышение температуры тела у пациентов с ВП $(\mathrm{p}<0,001)$ и частые ознобы $(\mathrm{p}<0,0001)$. Также у больных с ВП чаще встречались боли в грудной клетке $(56,5 \%$ vs $15,0 \%, \mathrm{p}<0,0001)$, цианоз $(43,5 \%$ vs $52 \%$, $\mathrm{p}=0,496)$, кровохарканье $(21,7 \%$ vs $3,0 \%, \mathrm{p}=0,006)$, снижение уровня сознания $(8,6 \%$ vs $9,0 \%, \mathrm{p}=0,663)$.

Также у пациентов с сочетанием ХОБЛ и ВП показатель СРБ сыворотки крови был выше, чем при отсутствии коморбидности $(105,8 \pm 66,1$ мг/л vs $28,7 \pm 19,7$ мг/л, p $<0,0001)$. Независимыми предикторами 30 -дневной летальности больных ХОБЛ с ВП стали уровень СРБ и индекс коморбидности Charlson. Таким образом определение уровня СРБ является высокочувствительным и специфичным тестом для диагностики бактериальной инфекции $>16,5$ мг/л и при ВП > 51,5 мг/л у больных ХОБЛ. При развитии осложнений ВП концентрации СРБ достоверно повышаются.

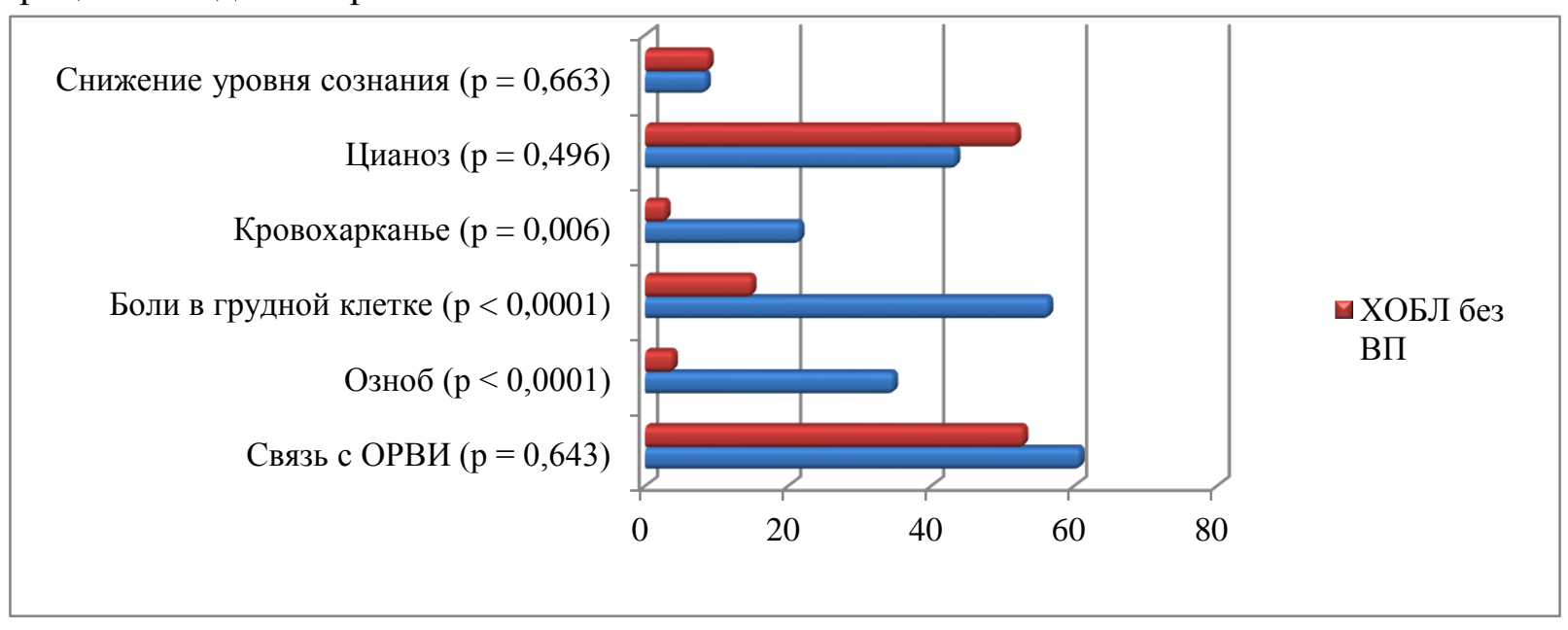

Рис. Клиника у пациентов с обострением ХОБЛ с развитием внебольничной пневмонии и без нее

[Баймакова и др., 2009]

Fig. Clinic in patients with exacerbation of COPD with the development of community-acquired pneumonia and without it [Baimakova et al., 2009] 
В более поздних исследованиях были установлены и другие параметры, которые можно использовать в диагностике и дифференциальной диагностике сложных ситуаций при сочетании ВП и ХОБЛ: прокальцитонин, фактор некроза опухоли- $\alpha$ и ИЛ-6 [Huerta et al., 2013].

\section{Клинический случай}

Больной А., 56 лет, поступил в пульмонологическое отделение ГБУ РО «Областная клиническая больница» 06.09.17 г. с жалобами: на одышку при незначительной нагрузке, кашель со слизисто-гнойной мокротой, ежедневные приступы удушья, чувство заложенности в грудной клетке, отеки голеней и стоп.

Анамнез заболевания: Длительное время страдает ХОБЛ, бронхиальной астмой. Постоянно принимает беклазон, беродуал, периодическими курсами ГКС. Ухудшение самочувствия почувствовал около 2 недель, когда появился кашель со слизисто-гнойной мокротой, усилилась одышка. За медицинской помощью не обращался, лечился самостоятельно. Принимал преднизолон 6 таблеток в день с постепенным снижением дозы до 2 таблеток в день, на фоне лечения самочувствие больного не улучшилось, кашель с отхождением слизисто-гнойной мокротой, усиливалась одышка. В связи с усиливающейся одышкой пациент вызвал бригаду скорой медицинской помощи и был доставлен в ГБУ РО «ОКБ» для дальнейшего обследования и лечения. В анамнезе хронический бескаменный холецистит. Хронический гепатит смешанной этиологии.

Данные физикального осмотра: Рост - 172 см, вес - 68 кг. Телосложение нормостеническое. Состояние средней степени тяжести. Кожные покровы бледной окраски, влажные. Наличие цианоза губ. Лимфотические узлы при пальпации не увеличены. Выраженные отеки стоп, голеней, больше слева.

В лёгких: дыхание везикулярное, сухие свистящие хрипы на выдохе, гудящие на вдохе и выдохе, в нижне-боковых отделах с обеих сторон. ЧДД - 26 в минуту, $\mathrm{SpO}_{2}-$ $80 \%$. Тоны сердца приглушены, ритм правильный с ЧСС 90 в минуту. АД 130/90 мм рт. ст. Живот мягкий, безболезненный. Печень пальпаторно не увеличена. Селезёнка не пальпируется. Физиологические отправления в норме.

Результаты дополнительного обследования:

На рентгенограмме легких от 06.09.17 г. - в прямой проекции легких определяется небольшой участок инфильтрации неоднородного характера слева в нижней доле. Справа без особенностей. Диафрагма, синусы просматриваются. Тень средостения не смещена. Заключение: очаговая пневмония слева.

На рентгенограмме легких от 12.09.17 г. - в прямой проекции по сравнению с рентгенограммой от 06.09.17 г. картина с отрицательной динамикой - объём инфильтрации слева увеличился.

РКТ легких от 19.09.17 г.: в легких дополнительных образований, теней очагового и инфильтративного характера не выявлено, определяются единичные спайки. Легочный рисунок перестроен в результате пневмосклероза, легочная ткань с признаками центрилобулярной эмфиземы. Трахея, главные, долевые сегментарные и субсегментарные бронхи прослеживаются, обычного диаметра, проходимы. Справа в нижней доле на уровне отхождения бронха $\mathrm{B}_{6}$ имеется деформация. Внутригрудные лимфатические узлы в небольшом количестве, в размерах не увеличены. Дополнительные образования в средостении не выявлены. Жидкости в плевральных полостях, в полости перикарда не выявлено. Костных деструктивных изменений нет. Заключение: Двусторонний диффузный пневмосклероз. Центрилобулярная эмфизема. Единичные спайки.

При поступлении были выполнены общий анализ крови, общий анализ мочи, коагулограмма, биохимический анализ крови, электрокардиограмма (ЭКГ).

Общий анализ крови: эритроциты - 4,4×10 12 лл; $\mathrm{Hb}-141$ г/л; лейкоциты $-8,1 \times 10^{9} /$ л; сегм - $79 \%$; лимфоциты - $16 \%$; моноциты - 5\%; СОЭ - 18 мм/ч. Общий анализ крови в 
динамике: эритроциты - 4,7×1012/л; Нb - 150 г/л; лейкоциты - 20,0×109/л; сегм - $92 \%$; лимфоциты - $5 \%$; моноциты - $3 \%$; СОЭ - 5 мм/ч.

Биохимический анализ крови: общий белок - 64 г/л; билирубин общий 11,1 мкмоль/л; билирубин прямой - 2,0 мкмоль/л; непрямой - 9,1 мкмоль/л; мочевина 7,3 ммоль/л, креатинин - 95 мкмоль/л, АЛТ - 16 Ед/л, АСТ - 16 Ед/л, остаточный азот 22,8 ммоль/л; глюкоза - 6,7 ммоль/л.

Коагулограмма: тромбиновое время - 13 сек; фибриноген - 5,4 г/л; АЧТВ 25,1 сек.; МНО - 0,97; протромбиновый индекс - 1,03; Д-димер - 1,4 мг/л.

ЭКГ - Умеренная синусовая тахикардия. Нормальное положение ЭОС. Нарушение внутрижелудочковой проводимости. Признаки увеличения левого предсердия.

УЗИ вен нижних конечностей: лимфостаз с обеих сторон.

Пациенту были назначены: дексаметазон 8 мг + эуфиллин 5,0 на 200 мл 0,9\% $\mathrm{NaCl}$ в/в кап., лазикс 0,5 в/в кап., лефлобакт 500 мг 2 раза/день в/в кап., метрогил 100 мг 2 раза/день, ингаляции беродуала, пульмикорта через небулайзер, сабокомб 250/100 2 вдоха 2 раза/день, омепразол, флюконазол 150 мг 1 раз/день.

На фоне проводимого лечения состояние пациента без значительной динамики, одышка и кашель сохраняются. 24.09.17 г. - в 09:50 состояние больного резко ухудшилось за счёт прогрессирующей дыхательной недостаточности. Было назначено: преднизолон 90 мг + эуфиллин 2,4\% - 5,0 + $\mathrm{NaCl} 0,9 \% 200$ мл в/в кап. Из-за гипотонии вызвана реанимационная бригада. На момент прихода бригады в 10:05 зафиксирована остановка сердечной и дыхательной деятельности. Начат непрямой массаж сердца с частотой 100 компрессий в минуту и ИВЛ мешком Амбу. В/в введено 90 мг преднизолона, адреналин 0,1 \%-1 мл каждую минуту. Несмотря на проведение реанимационных мероприятий, констатировали биологическую смерть.

Заключительный клинический диагноз:

Основное заболевание: ХОБЛ IV ст., обострение. Бронхиальная астма смешанная форма, тяжелое течение, гормонозависимая, неконтролируемая.

Осложнение основного заболевания: Пневмония слева в нижней доле средней степени тяжести. ДН ІІІ ст. Хроническое легочное сердце, декомпенсация.

Сопутствующие заболевания: ИБС: стенокардия напряжения III ФК. Гипертоническая болезнь III ст., 2 ст., риск 4 (очень высокий). ХСН ІІБ ст., ІІІ ФК.

По данным патолого-анатомического вскрытия: признаков за пневмонию не обнаружено и в заключительном патолого-анатомическом диагнозе пневмония не фигурирует.

\section{Выводы}

Целесообразность внесения ХОБЛ в список факторов риска ВП подтверждается высокой частотой встречаемости сопутствующей ХОБЛ у пациентов, госпитализированных по поводу ВП (до 62 \%). Большинство публикаций по исследуемой коморбидности включает отдельные клинические случаи тяжелых обострений ХОБЛ, сопровождающихся выраженной дыхательной недостаточностью, при этом из поля зрения исследователей выпадают пациенты с ВП на фоне легкой и средне-тяжелой ХОБЛ.

Клиническая диагностика ВП на фоне ХОБЛ и в наше время остается сложной задачей - частота постановки правильного прижизненного диагноза ВП у данной группы пациентов составляет менее $70 \%$ в РФ. Наличие ХОБЛ в анамнезе существенно влияет на процесс разрешения пневмонии, способствует гиперреактивности бронхов и более затяжному течению воспалительного процесса в легочной ткани по сравнению с пациентами с нормальной реактивностью бронхов. Кроме того, ХОБЛ как фоновое заболевание при ВП увеличивает вероятность летального исхода, что объясняется более выраженной обструкцией, дыхательной недостаточностью и высоким числом полигенных заболеваний у таких больных. 
Данный клинический случай иллюстрирует сложность диагностики и адекватного лечения пациентов с ХОБЛ и сочетанной патологией сердечно-сосудистой системы и своевременной и корректной диагностики осложнений, в том числе ВП. Трудности диагностики ВП у данного пациента возникли из-за наличия сопутствующего заболевания, при котором клинического проявления воспалительного процесса в легких маскируются симптомами фонового заболевания. При наличии в анамнезе сопутствующей ХОБЛ клинические и лабораторные критерии ВП (лихорадка, кашель с гнойной мокротой, усиление одышки, лейкоцитоз и т. д.), с одной стороны, приобретают неспецифичный характер, а с другой стороны - вписываются в картину обострения ХОБЛ.

\section{Список литературы}

1. Авдеев С.Н., Баймаканова Г.Е., Зубаирова П.А., Чучалин А.Г. 2006. Пневмония как причина острой дыхательной недостаточности у больных ХОБЛ. Пульмонология, 5: 115-119.

2. Баймакова Г.Е., Зубаирова П.А., Авдеев С.Н., Чучалин А.Г. 2009. Особенности клинической картины и течения внебольничной пневмонии у пациентов с хронической обструктивной болезнью легких. Пульмонология, 2: 33-41.

3. Биличенко Т.Н., Чучалин А.Г. 2018. Заболеваемость и смертность населения России от острых респираторных вирусных инфекций, пневмонии и вакцинопрофилактика. Терапевтический архив, 1: 22-26.

4. Внебольничная пневмония: клинические рекомендации. 2018. 98 с.

5. Дворецкий Л.И. 2015. Внебольничная пневмония у больных хронической обструктивной болезнью легких. Практическая пульмонология, 2: 17-24.

6. Ефремова О.А., Камышникова Л.А., Шелякина Е.В., Шкилёва И.Ю., Ходош Э.М., Ефименко Е.В. Роль хронической обструктивной болезни лёгких в развитии полиморбидной патологииНаучный результат. Медицина и фармация. 2016. 2. (4): 13-18.

7. Игнатьев В.А., Киселева Е.А., Зарембо И.А., Цветкова Л.Н., Кочергина Т.А., Конев В.Г., Пендюрин А.К. 2008. Пневмония у пациентов пожилого и старческого возраста. Клиническая геронтология, 6: 27-29.

8. Коррейа Л.Л., Лебедев Т.Ю., Ефремова О.А., Прощаев К.И., Литовченко Е.С. 2013. Проблема полиморбидности при сочетании хронической обструктивной болезни легких и некоторых сердечно-сосудистых заболеваний. Научные ведомости Белгородского государственного университета. Серия: Медицина. Фармация. 4: 12-17.

9. Кузубова Н.А., Титова О.Н., Волчков В.А., Козырев А.Г. 2014. Особенности внебольничной пневмонии у пациентов с хронической обструктивной болезнью легких. Трудный пациент, 3: 44-47.

10. Черняев А.Л. 2005. Диагностические ошибки в пульмонологии. Пульмонология, 3: 5-12.

11. Чучалин А.Г. 2016. Российское респираторное общество. Федеральные клинические рекомендации по диагностике и лечению хронической обструктивной болезни легких. М.: 68

12. Ewig S., Bauer T., Hasper E., Pizulli L., Kubini R., Luderitz B. 1995. Prognostic analysis and predictive rule for outcome of hospital-treated community-acquired pneumonia. Eur. Respir. J., 8: 392-397.

13. Farr B.M., Sloman A.J., Fisch M.J. 1991. Predicting death in patients hospitalized for community acquired pneumonia. Ann. Intern. Med., 115: 428-436.

14. GOLD. Global Initiative for chronic obstructive Lung Disease. Global strategy for the diagnosis, management, and prevention of chronic obstructive pulmonary disease. NHLBI/WHO workshop report. Update 2019 // www.goldcopd.com.

15. Groenewegen K.H., Schols A.M., Wouters E.F. 2003. Mortality and mortality-related factors after hospitalization for acute exacerbation of COPD. Chest., 124(2): 459-467.

16. Huerta A., Crisafulli E., Mene'ndez R., Martínez R., Soler N., Guerrero M., Montull B., Torres A. 2013. Pneumonic and non-pneumonic exacerbations of COPD: inflammatory response and clinical characteristics. Chest., 144: 1134-1142.

17. Lieberman D., Gelfer Y., Varshavsky R., Dvoskin B., Leinonen M., Friedman M.G. 2002. Pneumonic vs nonpneumonic acute exacerbations of COPD. Chest., 122(4): 1264-1270. 
18. Lin S.H., Ji BC., Shih Y.M., Chen C.H., Chan P.C., Chang Y.J., Lin Y.C., Lin C.H. 2013. Comorbid pulmonary disease and risk of community-acquired pneumonia in COPD patients. Int. J. Tuberc. Lung Dis., 17: 1638.

19. Loke Y.K., Kwok C.S., Wong J.M., Sankaran P., Myint P.K. 2013. Chronic obstructive pulmonary disease and mortality from pneumonia: meta-analysis. Int. J. Clin. Pract., 67 (5): 477-487.

20. Mannino D.M., Davis K.J., Kiri V.A. 2009. Chronic obstructive pulmonary disease and hospitalizations for pneumonia in a US cohort. Respir. Med., 103: 224-229.

21. Merino-Sánchez, Alfageme-Michavila I., Lima-Álvarez J. 2005. Prognosis in Patients With Pneumonia and Chronic Obstructive Pulmonary Disease Arch Bronconeumol, 41: 607-611.

22. Müllerova H., Chigbo C., Hagan G.W., Woodhead M.A., Miravitlles M., Davis K.J., Wedzicha J.A. 2012. The natural history of communityacquired pneumonia in COPD patients: a population database analysis. Respir Med, 106: 1124-33.

23. Rello J., Rodriguez A., Torres A., Roig J., Sole-Violan J., Garnacho-Montero J., de la Torre M.V., Sirvent J.M., Bodi M. 2006. Implications of COPD in patients admitted to the intensive care unit by community-acquired pneumonia. Eur. Respir. J., 27: 1210.

24. Restrepo M.I., Mortensen E.M., Pugh J.A., Anzueto A. 2006. COPD is associated with increased mortality in patients with community-acquired pneumonia. Eur. Respir. J., 28: 346-351.

25. Seneff M.G., Wagner D.P., Wagner R.P., Zimmerman J.E., Knaus W.A. 1995. Hospital and 1-year survival of patients admitted to intensive care units with acute exacerbation of chronic obstructive pulmonary disease. JAMA, 274: 1852-1857.

26. Sibila O. 2014. Prior cardiovascular disease increases long-term mortality in COPD patients with pneumonia. Eur. Respir. J., 43(1): 36-42.

\section{References}

1. Avdeev S.N., Bajmakanova G.E., Zubairova P.A., Chuchalin A.G. 2006. Pnevmoniya kak prichina ostroj dyhatel'noj nedostatochnosti u bol'nyh HOBL [Pneumonia as a factor of acute respiratory failure in patients with COPD]. Pul'monologiya, 5: 115-119.

2. Bajmakova G.E., Zubairova P.A., Avdeev S.N., CHuchalin A.G. 2009. Osobennosti klinicheskoj kartiny i techeniya vnebol'nichnoj pnevmonii u pacientov s hronicheskoj obstruktivnoj bolezn'yu legkih [Features of the clinical picture and course of community-acquired pneumonia in patients with chronic obstructive pulmonary disease]. Pul'monologiya, 2: 33-41.

3. Bilichenko T.N., Chuchalin A.G. 2018. Zabolevaemost' i smertnost' naseleniya Rossii ot ostryh respiratornyh virusnyh infekcij, pnevmonii i vakcinoprofilaktika [Morbidity and mortality of the Russian population from acute respiratory viral infections, pneumonia and vaccine prophylaxis]. Terapevticheskij arhiv, 1: 22-26.

4. Vnebol'nichnaya pnevmoniya: klinicheskie rekomendacii [Community-acquired pneumonia: clinical guidelines]. 2018. $98 \mathrm{~s}$.

5. Dvoreckij L.I. 2015. Vnebol'nichnaya pnevmoniya u bol'nyh hronicheskoj obstruktiynoj bolezn'yu legkih [Community-acquired pneumonia in patients with chronic obstructive pulmonary disease]. Prakticheskaya pul'monologiya, 2: 17-24.

6. Efremova O.A., Kamyshnikova L.A., Sheljakina E.V., Shkiljova I.Ju., Hodosh Je.M., Efimenko E.V. Rol' hronicheskoj obstruktivnoj bolezni ljogkih v razvitii polimorbidnoj patologii [The role of chronic obstructive pulmonary disease in the development of polymorbidity pathology]. Nauchnyj rezul'tat. Medicina i farmacija. 2016. 2. (4): 13-18.

7. Ignat'ev V.A., Kiseleva E.A., Zarembo I.A., Cvetkova L.N., Kochergina T.A., Konev V.G., Pendyurin A.K. 2008. Pnevmoniya u pacientov pozhilogo i starcheskogo vozrasta. Some aspects of the development of pneumonia against the background of chronic obstructive pulmonary disease in the elderly]. Klinicheskaya gerontologiya, 6: 27-29.

8. Korreja L.L., Lebedev T.Yu., Efremova O.A., Proshchaev K.I., Litovchenko E.S. 2013. Problema polimorbidnosti pri sochetanii hronicheskoj obstruktivnoj bolezni legkih i nekotoryh serdechno-sosudistyh zabolevanij [The problem of polymorbidity of chronic obstructive pulmonary disease and some cardiovascular diseases]. Nauchnye vedomosti Belgorodskogo gosudarstvennogo universiteta. Seriya: Medicina. Farmaciya. 4: 12-17.

9. Kuzubova N.A., Titova O.N., Volchkov V.A., Kozyrev A.G. 2014. Osobennosti vnebol'nichnoj pnevmonii u pacientov s hronicheskoj obstruktivnoj bolezn'yu legkih [Features of community-acquired pneumonia in patients with chronic obstructive pulmonary disease]. Trudnyj pacient, 3: 44-47. 
10. Chernyaev A.L. 2005. Diagnosticheskie oshibki v pul'monologii [Diagnostic mistakes in pulmonology]. Pul'monologiya, 3: 5-12.

11. Chuchalin A.G. 2016. Rossijskoe respiratornoe obshchestvo. Federal'nye klinicheskie rekomendacii po diagnostike i lecheniyu hronicheskoj obstruktivnoj bolezni legkih [Russian Respiratory Society Federal clinical guidelines for the diagnosis and treatment of chronic obstructive pulmonary disease]. M.: 68

12. Ewig S., Bauer T., Hasper E., Pizulli L., Kubini R., Luderitz B. 1995. Prognostic analysis and predictive rule for outcome of hospital-treated community-acquired pneumonia. Eur. Respir. J., 8: 392-397.

13. Farr B.M., Sloman A.J., Fisch M.J. 1991. Predicting death in patients hospitalized for community acquired pneumonia. Ann. Intern. Med., 115: 428-436.

14. GOLD. Global Initiative for chronic obstructive Lung Disease. Global strategy for the diagnosis, management, and prevention of chronic obstructive pulmonary disease. NHLBI/WHO workshop report. Update 2019 // www.goldcopd.com.

15. Groenewegen K.H., Schols A.M., Wouters E.F. 2003. Mortality and mortality-related factors after hospitalization for acute exacerbation of COPD. Chest., 124 (2): 459-467.

16. Huerta A., Crisafulli E., Mene'ndez R., Martínez R., Soler N., Guerrero M., Montull B., Torres A. 2013. Pneumonic and non-pneumonic exacerbations of COPD: inflammatory response and clinical characteristics. Chest., 144: 1134-1142.

17. Lieberman D., Gelfer Y., Varshavsky R., Dvoskin B., Leinonen M., Friedman M.G. 2002. Pneumonic vs nonpneumonic acute exacerbations of COPD. Chest., 122(4): 1264-1270.

18. Lin S.H., Ji B.C., Shih Y.M., Chen C.H., Chan P.C., Chang Y.J., Lin Y.C., Lin C.H. 2013. Comorbid pulmonary disease and risk of community-acquired pneumonia in COPD patients. Int. J. Tuberc. Lung Dis., 17: 1638.

19. Loke Y.K., Kwok C.S., Wong J.M., Sankaran P., Myint P.K. 2013. Chronic obstructive pulmonary disease and mortality from pneumonia: meta-analysis.Int. J. Clin. Pract., 67 (5): 477-487.

20. Mannino D.M., Davis K.J., Kiri V.A. 2009. Chronic obstructive pulmonary disease and hospitalizations for pneumonia in a US cohort. Respir. Med., 103: 224-229.

21. Merino-Sánchez, Alfageme-Michavila I., Lima-Álvarez J. 2005. Prognosis in Patients With Pneumonia and Chronic Obstructive Pulmonary Disease Arch Bronconeumol, 41: 607-611.

22. Müllerova H., Chigbo C., Hagan G.W., Woodhead M.A., Miravitlles M., Davis K.J., Wedzicha J.A. 2012. The natural history of communityacquired pneumonia in COPD patients: a population database analysis. Respir Med, 106: 1124-33.

23. Rello J., Rodriguez A., Torres A., Roig J., Sole-Violan J., Garnacho-Montero J., de la Torre M.V., Sirvent J.M., Bodi M. 2006. Implications of COPD in patients admitted to the intensive care unit by community-acquired pneumonia. Eur. Respir. J., 27: 1210.

24. Restrepo M.I., Mortensen E.M., Pugh J.A., Anzueto A. 2006. COPD is associated with increased mortality in patients with community-acquired pneumonia. Eur. Respir. J., 28: 346-351.

25. Seneff M.G., Wagner D.P., Wagner R.P., Zimmerman J.E., Knaus W.A. 1995. Hospital and 1-year survival of patients admitted to intensive care units with acute exacerbation of chronic obstructive pulmonary disease. JAMA, 274: 1852-1857.

26. Sibila O. 2014. Prior cardiovascular disease increases long-term mortality in COPD patients with pneumonia. Eur. Respir. J., 43(1): 36-42.

\section{Ссылка для цитирования статьи For citation}

Урясьев О.М., Панфилов Ю.А., Гринькова Я.Н., Гранаткин М.А., Пыко А.А. 2020. Внебольничная пневмония у пациентов с ХОБЛ: временная коморбидность или фактор риска летального исхода? Актуальные проблемы медицины, 43(1): 28-37. DOI

Uryasyev O.M., Panfilov Yu.A., Grinkova Y.N., Granatkin M.A., Pyko A.A. 2020. Communityacquired pneumonia in patients with COPD:temporary comorbidity or risk factor for death? Challenges in Modern Medicine, 43(1): 28-37 (in Russian). DOI 